\title{
Proposta de melhorias na gestão de empresas de construção civil: um estudo de caso internacional
}

\author{
Proposal of improvements in the management of construction companies: an \\ international case study
}

\section{Propuesta de mejoras en la gestión de empresas de construcción civil: un estudio de caso internacional}

\author{
Joaquim Teixeira Netto ${ }^{1}$ \\ João Alberto Neves ${ }^{1}$ \\ Walter Passos Filho ${ }^{1}$ \\ Nylvandir Liberato Oliveira ${ }^{1}$
}

Recebido em: 11/06/2018. Revisado e aprovado em: 14/04/2019. Aceito em: 22/05/2019.

DOI: http://dx.doi.org/10.20435/inter.v21i3.2042

\begin{abstract}
Resumo: A construção civil tem um grande impacto no desenvolvimento local. Entretanto verifica-se que as empresas de construção apresentam, em geral, gestão deficiente. Este artigo tem por objetivo identificar as lacunas na gestão de empresas da construção civil e propor melhorias. Inicialmente, foi realizada uma revisão da literatura com a identificação dos problemas na gestão da construção; em seguida, foi realizada uma pesquisa em empresas de construção civil do Brasil, Índia, México, Ucrânia e Alemanha. Finalmente, foram propostas melhorias na gestão da construção civil, baseadas nas melhores práticas de diferentes países. Palavras-chave: metodologia de gestão; gestão enxuta; construção civil; desenvolvimento local.
\end{abstract}

Abstract: Civil construction has a major impact on local development. However, it can be seen that construction companies, in general, have poor management. This article aims to identify gaps in the management of construction companies and propose improvements. Initially, we carried out a literature review with the identification of problems in construction management, followed by a survey of construction companies in Brazil, India, Mexico, Ukraine, and Germany. Finally, we proposed improvements in the management of civil construction, based on the best practices of different countries.

Keywords: methodology of management; lean management; civil construction; local development.

Resumen: La construcción civil tiene un gran impacto en el desarrollo local. Sin embargo, se verifica que las empresas de construcción presentan, en general, una gestión deficiente. Este artículo tiene por objetivo identificar las lagunas en la de gestión de empresas de la construcción civil y proponer mejoras. En el presente trabajo, se realizó una revisión de la literatura con la identificación de los problemas en la gestión de la construcción; en seguida, se realizó una investigación en empresas de construcción civil de Brasil, India, México, Ucrania y Alemania. Finalmente, se propusieron mejoras en la gestión de la construcción, basadas en las mejores prácticas de diferentes países.

Palabras clave: metodología de gestión; gestión de la construcción; construcción civil; desarrollo local.

\section{INTRODUÇÃO}

No Brasil, a construção civil apresenta um grande impacto no desenvolvimento local e no PIB regional. No estado do Rio de Janeiro, foi verificado que o setor da construção civil apresentou entre 2008/2012 uma taxa de crescimento de 34,7\%, o que representou 4,5\% do PIB do estado e $3 \%$ do país (FIRJAN, 2014). Apesar da importância do setor, verifica-se que ele apresenta baixa produtividade e qualidade (FREITAG, 2015). Foi identificado pela Firjan (2014) que o principal problema se refere a deficiências na utilização de metodologias de gestão e na utilização de ferramentas de TI.

Obras de construção civil apresentam um enorme desperdício e uma baixa eficiência quando comparadas com outras indústrias. Prejuízos gerados em obras de infraestrutura no 
mundo geram perda de 1 trilhão de dólares globalmente (DOBBS; POHL; LIN, 2013). Os atrasos e aumentos frequentes de custo na construção civil foram os grandes impulsionadores para que as empresas passassem a direcionar um maior esforço no planejamento e no monitoramento dos projetos de construção. A gestão de projetos de construção enfrenta dificuldades, sendo comum observar orçamentos excedidos, atrasos de cronograma, baixa qualidade e baixa produtividade no processo construtivo (ARROTÉIA; AMARAL; MELHADO, 2014). Alguns autores consideram que esse fato se deve à natureza complexa dos empreendimentos de construção (HORSTMAN; WITTEVEEN, 2013). A gestão de projetos associada a sistemas de medição de desempenho tem sido proposta por diferentes autores, em especial por intermédio de indicadores de desempenho (BABAR; THAHEEM; AYUB, 2017).

A proposta da pesquisa foi avaliar a gestão da construção civil mostrando as lacunas nos modelos de gestão atual e propondo um novo modelo de gestão. Inicialmente, foi realizada uma revisão da literatura sobre as metodologias de gestão e suas lacunas; posteriormente, foram feitas entrevistas em empresas de construção civil do Brasil, Índia, México, Ucrânia e Alemanha. A pesquisa é inédita, pois busca identificar os principais problemas referentes à baixa eficiência e produtividade, detectando os motivos centrais para os atrasos e aumentos de custo frequentes na construção civil, bem como as lacunas nas metodologias de gestão da construção, e propor possíveis melhorias na gestão da construção relativa ao seu planejamento e monitoramento nos aspectos de prazo e custo. Além disso, foram propostas melhorias na gestão da construção por meio da integração de diferentes metodologias de gestão baseadas em experiências observadas em diferentes países.

\section{PROBLEMAS DE GESTÃO EM EMPRESAS NA CONSTRUÇÃO CIVIL}

Na literatura, verifica-se que as principais causas dos problemas na construção civil se devem a falhas de gestão. Arrotéia, Amaral e Melhado (2014) identificaram problemas relacionados às pessoas, tecnologias e dados envolvidos no projeto, que resultam em atrasos nas obras. Segundo os autores, a indústria da construção se caracteriza por ter uma estrutura organizacional excessivamente fragmentada, devendo o coordenador promover a integração das equipes de planejamento, com o apoio de ferramentas de TI.

Pesquisas recentes sobre os problemas na gestão na construção civil abordam as deficiências na integração do cronograma com o custo, dificuldades nos processos de medição, falta de treinamento da equipe, estrutura organizacional inadequada e excessivamente funcional e a falta de uso de sistemas adequados de Tecnologia da Informação (TI) (NETTO et al., 2014). Os problemas de atrasos se referem em grande parte a questões internas da empresa relativas à qualificação de pessoas, metodologias, sistemas de gestão e planejamento nas obras (DE FILIPPI; MELHADO, 2015). Em avaliação da Firjan, foi identificado que o principal problema na construção civil se refere a falhas na gestão e à necessidade de intensificar o uso de novas tecnologias (FIRJAN, 2014). Autores como Grau et al. (2014) corroboram esse fato e acrescentam que o desempenho da construção civil deve considerar um conjunto de fatores, como: aspectos técnicos, de gestão e sociais. Problemas relacionados à falta de planejamento, falta de metodologia de gestão, obras atrasadas e projetos concluídos com valores muito acima do orçamento são frequentes na construção civil (NAZÁRIO; AZEVEDO, 2016).

Os problemas da construção civil podem ser classificados nas perspectivas de estrutura organizacional, metodologia de gestão, pessoas e tecnologia da informação (ANDÚJAR-MONTOYA 
et al., 2015). A figura 1 resume os tipos de problemas de gestão encontrados na indústria da construção.

Figura 1 - Problemas de gestão na construção civil

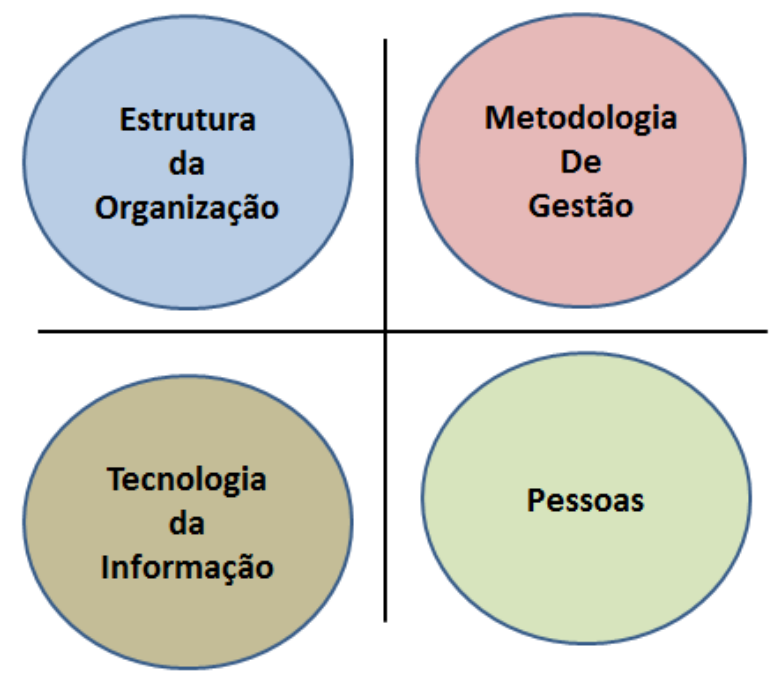

Fonte: Adaptado de Andújar-Montya et al. (2015).

Na Figura 1, observam-se os diferentes aspectos relacionados a pessoas, metodologias, estrutura da organização e tecnologia da informação. As metodologias de gestão correspondem a um conjunto de processos da empresa; tecnologia da informação são os sistemas que dão suporte aos processos; pessoas são os colaboradores da empresa; e a estrutura da organização são os departamentos da empresa e sua organização.

O resumo dos problemas identificados nas empresas de construção se encontra no Quadro 1 abaixo.

Quadro 1 - Resumo dos problemas de gestão identificados na construção civil

\begin{tabular}{|l|l|l|}
\hline \multicolumn{1}{|c|}{ Tipo } & \multicolumn{1}{|c|}{ Descrição } & \multicolumn{1}{c|}{ Ref. } \\
\hline Pessoas & $\begin{array}{l}\text { Ausência de qualificação de pessoas. Falta de integração das } \\
\text { equipes. }\end{array}$ & $\begin{array}{l}\text { Arrotéia, Amaral e } \\
\text { Melhado (2014), } \\
\text { Netto et al. (2015) }\end{array}$ \\
\hline $\begin{array}{l}\text { Estrutura } \\
\text { Organizacional }\end{array}$ & $\begin{array}{l}\text { Estrutura organizacional inadequada, fragmentada e } \\
\text { excessivamente funcional. }\end{array}$ & $\begin{array}{l}\text { Netto et al. (2015), } \\
\text { Arrotéia, Amaral e } \\
\text { Melhado (2014) }\end{array}$ \\
\hline $\begin{array}{l}\text { Tecnologia } \\
\text { da Informação }\end{array}$ & $\begin{array}{l}\text { Uso inadequado ou falta de uso de sistemas de TI (Tecnologia } \\
\text { da Informação) na gestão da construção civil. Apoio na } \\
\text { integração das pessoas por uma ferramenta de Tl. }\end{array}$ & $\begin{array}{l}\text { Firjan (2014), Netto } \\
\text { et al. (2015) }\end{array}$ \\
\hline $\begin{array}{l}\text { Metodologia de de } \\
\text { Gestão }\end{array}$ & $\begin{array}{l}\text { Metodologia de gestão e planejamento deficiente das obras. } \\
\text { Deficiências na integração do cronograma com o custo e } \\
\text { dificuldades nos processos de medição. }\end{array}$ & $\begin{array}{l}\text { Firjan (2014), De } \\
\text { Filippi e Melhado } \\
\text { (2015), Nazário e } \\
\text { Azevedo (2016) }\end{array}$ \\
\hline
\end{tabular}

Fonte: Os próprios autores.

Pode-se verificar no Quadro 1 o resumo dos principais problemas identificados na 
construção civil. A baixa eficiência e produtividade é citada por diferentes autores, tendo como origem: estrutura organizacional, integração das equipes, falta de uso de ferramenta de TI adequada, falta de planejamento, falta do uso de metodologias de gestão e falta de comunicação.

\section{METOdOLOGIA DE GESTÃO DO PMBOK}

O guia PMBOK (PROJECT MANAGEMENT INSTITUTE [PMI], 2017) é publicado pelo PMI e agrega um conjunto de conhecimento de gestão já consagrado por práticas de mercado. $\mathrm{O}$ controle do prazo do projeto ocorre a partir da confecção do cronograma, com a divisão da obra (escopo do projeto) em uma Estrutura Analítica de Projeto (EAP) ou pacotes de trabalho. Os pacotes de trabalho são então divididos em atividades que são sequenciadas. O sequenciamento dos pacotes de trabalho auxilia na determinação do tempo, que, após o sequenciamento, é determinado pelo Método do Caminho Crítico ou Critical Path Method (CPM), que é utilizado em mais de 90\% dos projetos nos Estado Unidos (LOWE et al., 2012). Outro aspecto importante é o custo nas obras obtido com a soma do custo do material, mão de obra e custos indiretos de cada atividade (NETTO et al., 2015).

Os processos para a obtenção de prazo, custo e gerenciamento por valor agregado (EVM) podem ser visualizados na Figura 2.

Figura 2 - Processos de Gestão: Cronograma, Custo, EVM e Risco

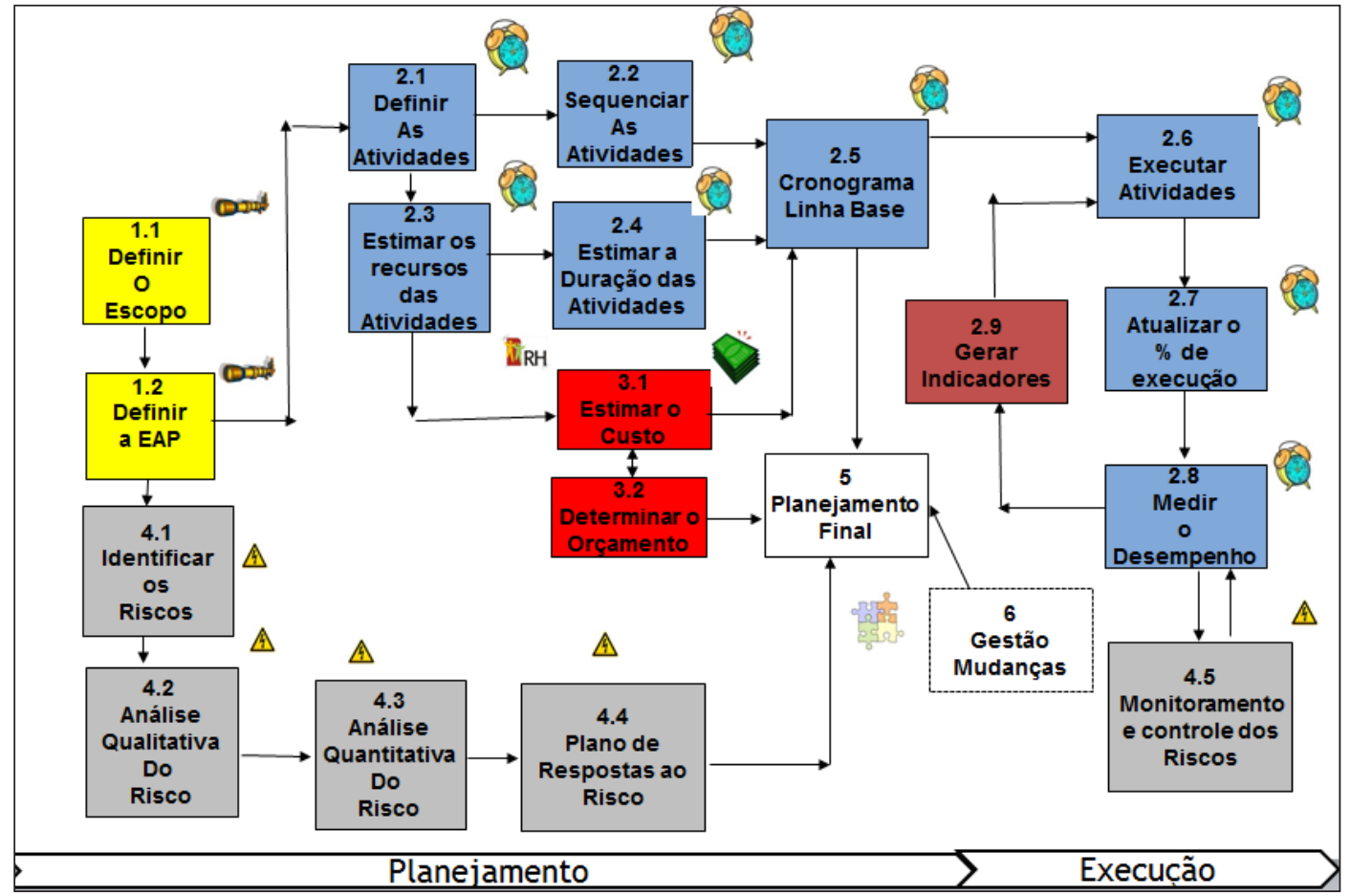

Fonte: Adaptado pelo autor, do PMBOK (PMI, 2017).

Na Figura 2, observam-se os processos de escopo (1), cronograma (2), custo (3) e risco (4). O ciclo de planejamento inicia-se com a definição do escopo (1.1); em seguida, a divisão do escopo em pacotes de trabalho (EAP) (1.2). A definição do cronograma inicia-se na definição das atividades (2.1); em seguida, é realizado o sequenciamento das atividades (2.2), com a definição de recursos por atividade (2.3), duração das atividades (2.4), datas de início e fim de cada atividade e restrições entre elas. A definição do orçamento é iniciada pelo processo de estimar o custo 
(3.1) e o plano de contas que permite a apropriação de custo para cada pacote de trabalho. O orçamento final (3.2) inclui os recursos materiais e humanos para cada atividade, os custos indiretos e ainda os fundos de reserva. O cronograma inicial ou linha-base (2.5) integra os custos e o prazo do projeto e será utilizado para medir a linha-base de desempenho, representando o final da fase de planejamento (5). O ciclo de execução inicia-se com a execução das atividades (2.6) e a atualização do percentual de execução, o custo e o prazo (2.7). Em seguida, a atividade de medir o desempenho (2.8) compara a previsão com a execução das atividades e obtém os indicadores (2.9). O controle de mudança é a formalização de possíveis mudanças que possam acontecer durante o projeto (6) (PMI, 2017).

O processo de riscos considera cinco atividades principais: identificação dos riscos (4.1), análise qualitativa dos riscos (4.2), análise quantitativa dos riscos (4.3), plano de resposta aos riscos (4.4) e monitoramento e controle dos riscos (4.5) (PMI, 2017). A identificação dos riscos é uma fase em que se definem as características essenciais dos riscos, a descrição, suas causas e consequências. A identificação dos riscos não é uma atividade simples e depende da opinião de especialistas (DE FILIPPI; MELHADO, 2015).

$\mathrm{Na}$ área de construção civil, a metodologia do PMBOK têm sido uma das mais utilizadas, apesar de necessitar de algumas adequações para a sua utilização na construção (SEPPÄNEN; EVINGER; MOUFLARD, 2014). Alguns autores ressaltam que a Gestão Enxuta apresenta um melhor controle da produção, redução dos desperdícios e melhoria na produtividade na construção (ANDÚJAR-MONTOYA et al., 2015; MELO; DESCHAMPS; DA COSTA, 2017; ZHANG, 2017). Segundo Koskela et al. (2014), a utilização de cronograma baseado no CPM apresenta dificuldades no acompanhamento no canteiro de obras e nas informações de longo prazo, sugerindo a utilização dos processos de gestão enxuta para a construção civil.

\section{METODOLOGIA DE GESTÃO ENXUTA}

O Lean Manufacturing foi originalmente criado pela Toyota ${ }^{\circ}$, durante o período de reconstrução do Japão após a Segunda Guerra Mundial. Os conceitos de manufatura enxuta (Lean Manufacturing) foram utilizados para a área de gestão e, atualmente, essa filosofia operacional é conhecida como gestão enxuta (Lean Management), sendo focada na redução dos desperdícios (ZHANG, 2017). A indústria da construção tem norteado diretrizes de projetos de gestão enxuta constituídos por organismos internacionais como o Lean Construction Institute (LCI). No Brasil, o Núcleo Orientado para a Inovação da Edificação (Norie) tem desenvolvido trabalhos sobre a utilização da gestão enxuta na construção civil (MOURA; FORMOSO, 2009).

O Last Planner System (LPS, Sistema do Último Planejador) utiliza o conceito de gestão enxuta de produção por meio da organização das tarefas para a indústria da construção civil (KIM; BALLARD, 2010). O último planejador é o responsável por organizar as tarefas de curto prazo a partir do fluxo de trabalho. O planejamento no LPS é desdobrado em três planos principais: longo, médio e curto prazo (MELO; DESCHAMPS; DA COSTA, 2017).

A Figura 3 exibe os planos principais. 


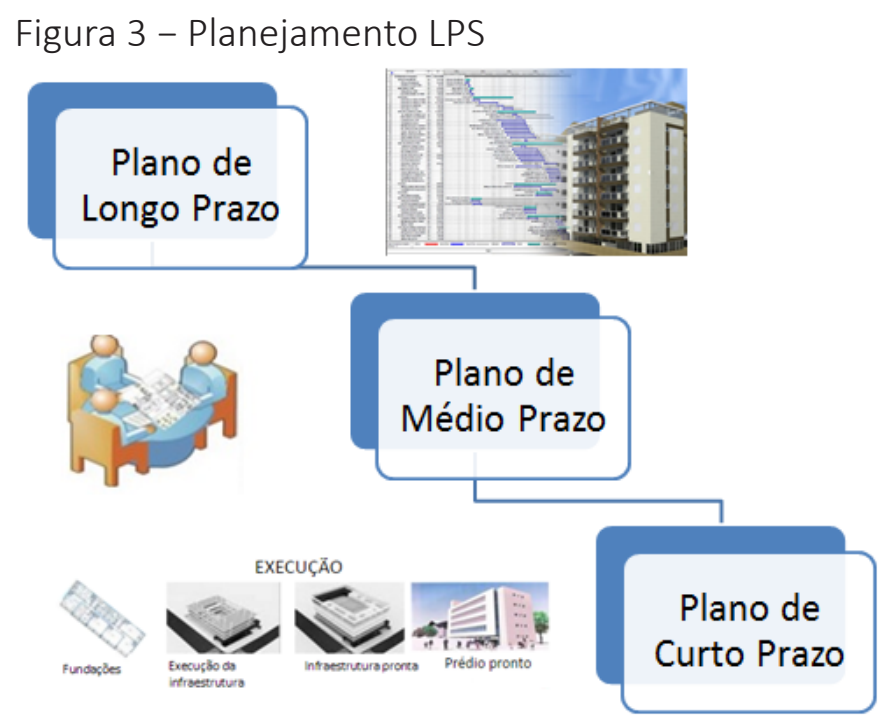

Fonte: Adaptado de Melo, Deschamps, Da Costa (2017).

Na Figura 3, observam-se os três planos do Sistema do Último Planejador (LPS): o Plano de Longo Prazo, também conhecido como plano mestre ou cronograma mestre com os marcos principais (milestone) da obra, as atividades e as tarefas com as respectivas etapas principais ou pacotes de trabalho; o Cronograma de Médio Prazo ou Look Ahead Plan (Plano para Olhar à Frente), que verifica o que foi realizado e as necessidades de material e pessoal para as próximas tarefas; e o Plano de Curto Prazo, que corresponde ao planejamento da produção no canteiro de obras e a execução direta das tarefas (make-ready process) (DANIEL et al., 2017).

O sistema LPS e o CPM são baseados em atividades (AB-Activity Based), entretanto as atividades em construção são realizadas em locais específicos dentro da obra. Sistemas baseados em localização facilitam esse processo e são designados de Locate Based System (LBS) (OLIVIERI; GRANJA; PICCHI, 2016). O sistema LBS permite um melhor planejamento e visualização da execução do trabalho, pois adiciona a localização e os recursos de acordo com o fluxo de trabalho, permitindo que a técnica do CPM possa ser mais bem utilizada (SEPPÄNEN; EVINGER; MOUFLARD, 2014).

O Planejamento Takt Time Planning (TTP) tem sua origem na expressão alemã "Takt", que significa cadência como o "tic-tac" de um relógio. Apesar de a expressão ser antiga para a utilização da manufatura na Alemanha, esse conceito na construção civil é relativamente recente, tendo sido aperfeiçoado por diferentes autores que buscam melhorar o fluxo de produção na construção civil (FRANDSON; BERGHEDE; TOMMELEIN, 2013).

Na revisão da literatura realizada, pode-se verificar que cada metodologia de gestão apresenta características próprias e lacunas que se encontram resumidas no Quadro 2. 
Quadro 2 - Características das metodologias na gestão da construção

\begin{tabular}{|c|c|c|}
\hline $\begin{array}{l}\text { Método de } \\
\text { Gestão }\end{array}$ & Características & Ref. \\
\hline $\begin{array}{l}\text { Gestão de } \\
\text { Cronograma } \\
\text { (CPM) }\end{array}$ & $\begin{array}{l}\text { Controla o prazo do projeto a partir de cada atividade } \\
\text { e relacionamento entre elas. Entretanto foram } \\
\text { observadas dificuldades no acompanhamento das } \\
\text { atividades no canteiro de obras. }\end{array}$ & PMI (2017) \\
\hline $\begin{array}{l}\text { Gestão por } \\
\text { Valor Agregado } \\
\text { (EVM) }\end{array}$ & $\begin{array}{l}\text { Acompanha o prazo, o custo e os desvios por meio do } \\
\text { uso de indicadores e pela visualização gráfica da curva } \\
\text { "S". É complexo, uma vez que envolve a integração dos } \\
\text { processos de custo, escopo e o prazo da obra. }\end{array}$ & $\begin{array}{l}\text { Netto et al. (2015), } \\
\text { Narbaev e De Marco } \\
\text { (2013) }\end{array}$ \\
\hline $\begin{array}{l}\text { Gestão de } \\
\text { Riscos }\end{array}$ & $\begin{array}{l}\text { Gerenciar os riscos e diminuir as incertezas do projeto. } \\
\text { A identificação dos riscos não é simples e depende } \\
\text { da opinião de especialistas e do histórico de obras } \\
\text { anteriores. }\end{array}$ & $\begin{array}{l}\text { Renuka, Umarani e } \\
\text { Kamal (2014). } \\
\text { Nasirzadeh, Khanzadi e } \\
\text { Rezaie (2014) }\end{array}$ \\
\hline $\begin{array}{l}\text { Last Planner } \\
\text { System (LPS) }\end{array}$ & $\begin{array}{l}\text { Acompanha o cronograma em planos de: longo prazo, } \\
\text { médio prazo e curto prazo. Não detalha a forma de } \\
\text { se obter melhorias no processo de produção, tendo } \\
\text { a necessidade de colaboração entre os diferentes } \\
\text { especialistas. }\end{array}$ & $\begin{array}{l}\text { Seppänen, Evinger, } \\
\text { Mouflard (2014) } \\
\text { Andújar Montoya et al. } \\
\text { (2015) } \\
\text { Melo; Deschamps e Da } \\
\text { Costa (2017) }\end{array}$ \\
\hline TTP & $\begin{array}{l}\text { Busca a melhoria dos processos de produção no } \\
\text { canteiro de obras, identificando o local das atividades } \\
\text { de produção. Deve haver colaboração entre os } \\
\text { diferentes especialistas. }\end{array}$ & $\begin{array}{l}\text { Frandson; Berghede e } \\
\text { Tommelein (2013) }\end{array}$ \\
\hline
\end{tabular}

Fonte: Os próprios autores.

No Quadro 2, são identificadas as características de diferentes metodologias utilizadas na gestão da construção civil e suas lacunas. Observa-se a necessidade de utilização de mais de uma metodologia na gestão da construção, pois algumas lacunas de uma determinada metodologia são preenchidas por outras metodologias.

Os aspectos relativos à metodologia de pesquisa se encontram descritas na próxima seção.

\section{MÉTODO DE PESQUISA}

A pesquisa foi do tipo exploratória, com um estudo de caso múltiplo com participantes de diferentes países. O estudo de caso iniciou-se em 2017, no Brasil, e posteriormente foi desenvolvido na Alemanha. Foram realizadas entrevistas semiestruturadas, observações diretas e análise documental em construtoras de pequeno, médio e grande porte em projeto de construção predial, shopping, condomínio, museu, hotel, laboratório. A amostra selecionada foi por conveniência, devido à disponibilidade de tempo, fácil acesso e desejo de participar da pesquisa (FARROKHI; MAHMOUDI-HAMIDABAD, 2012), além disso, buscou-se uma diversificação da amostra conforme sugerido por Gil (2002), com projetos de construção de diferentes tamanhos nas áreas púbicas e privadas no Brasil e no exterior. No Quadro 3, encontram-se resumidas as categorias das empresas que foram utilizadas na pesquisa. 
Quadro 3 - Síntese descritiva da amostra

\begin{tabular}{|c|c|c|c|c|c|c|c|}
\hline Item & Empresa & $\begin{array}{c}\text { Tipo de } \\
\text { Construção }\end{array}$ & $\begin{array}{c}\text { Tamanho do } \\
\text { Empreendimento }\end{array}$ & $\begin{array}{c}\text { Tamanho } \\
\text { da Empresa }\end{array}$ & $\begin{array}{c}\text { Tipo de } \\
\text { Cliente }\end{array}$ & País & $\begin{array}{c}\text { Fontes } \\
\text { de Evidência }\end{array}$ \\
\hline 1 & D & Prédio & Médio & Média & Privado & Brasil & $(1)(2)(3)$ \\
\hline 2 & E & $\begin{array}{c}\text { Laboratório, } \\
\text { Hospital }\end{array}$ & Grande & Grande & Autarquia & Brasil & $(1)(2)(3)$ \\
\hline 3 & F & Hotel & Grande & Pequena & Privado & Índia & $(1)$ \\
\hline 4 & G & Shopping & Grande & Pequena & Privado & México & $(1)$ \\
\hline 5 & H & $\begin{array}{c}\text { Condomínio } \\
\text { Residencial }\end{array}$ & Médio & Pequena & Privado & Ucrânia & $(1)$ \\
\hline 6 & I & Museu & Grande & Grande & Privado & Alemanha & $(1)(4)$ \\
\hline
\end{tabular}

Fonte: Os próprios autores.

Pode-se verificar no Quadro 3 que a amostra é bem diversificada, apresentando empreendimentos de diversos tipos no Brasil, Índia, Ucrânia, México e Alemanha em projetos de construção. As fontes de evidência foram classificadas em (1) entrevista semiestruturada, (2) observação direta, análise documental na empresa (3) e análise documental no website da empresa (4).

\section{ESTUDO DE CASO}

\section{Brasil}

O estudo de caso iniciou-se no Brasil no estado do Rio de Janeiro, com uma entrevista com uma construtora privada (empresa "D") que atua em construção de prédios residenciais na cidade de Niterói. Foi entrevistado o diretor técnico, que informou que utiliza apenas o cronograma para o controle das obras, entretanto têm ocorrido dificuldades na sua atualização devido ao acompanhamento das tarefas no canteiro de obras. Em relação às ferramentas de gestão de Risco, EVM e Lean, informou que não utiliza nos projetos, devido à falta de conhecimento dessas ferramentas.

A segunda empresa entrevistada foi uma organização governamental (empresa " $E$ "), com obras em laboratórios e hospitais em diferentes estados. Foi identificado que a empresa utiliza para a gestão dos projetos o cronograma físico e financeiro e a curva "S" (EVM) sem utilização dos indicadores, como tampouco utiliza a gestão de risco e a gestão enxuta nos projetos. Foi observada baixa maturidade em gestão de projetos e dificuldades em atualização do EVM.

A segunda etapa da pesquisa de campo ocorreu na Alemanha, onde foram entrevistados gerentes de projeto dos seguintes países: Índia, México, Ucrânia e Alemanha.

\section{Índia}

O gerente de projeto da empresa " $F$ " foi perguntado sobre a ocorrência de atrasos nos projetos de construção. Ele informou que os atrasos são frequentes. Em relação às ferramentas de gestão, afirmou que o controle de custo é feito no MsExcell ${ }^{\circ}$ e o cronograma no MsProject ${ }^{\circ}$. E, em relação ao controle de risco e à gestão enxuta, informou que, em geral, esses não são utilizados. 


\section{México}

O entrevistado da empresa " $G$ " informou que utiliza o cronograma físico e financeiro para o controle de prazo e custo, e o EVM, para o seu acompanhamento. Sob o aspecto da gestão do tempo do projeto, foi relatado que os atrasos na construção no México ocorrem com frequência. O controle de risco não é feito, mas o entrevistado citou como exemplo a obra de um shopping, em que a instabilidade política provocou aumento de material e mão de obra. Afirmou, ainda, que a abordagem Lean não é utilizada nos projetos.

\section{Ucrânia}

O gerente da empresa " $\mathrm{H}$ " informou que controla as obras apenas com um cronograma no MsProject ${ }^{\circ}$. Informou que tem feito obras em condomínios residenciais na cidade de Smila, na Ucrânia. O controle de risco não é feito, mas considera como maior risco a instabilidade política e econômica. O EVM e a abordagem Lean não são utilizados nos projetos.

\section{Alemanha}

O entrevistado é gerente de projetos de uma empresa de construção (empresa "I"), com 2.400 funcionários e sede na cidade de Stuttgart, capital do estado de Baden-Württemberg, na Alemanha. Em relação às principais obras realizadas, foi citado o Museu da Mercedes em Stuttgart (Alemanha). O controle de risco é iniciado juntamente da concepção da obra, em que são identificados os fatores de risco; posteriormente, é feito o acompanhamento mensal juntamente do cliente e demais empresas que participam da obra. Em relação à utilização da ferramenta de EVM, o entrevistado informou que esse gerenciamento é feito e atualizado mensalmente por meio de relatórios de gestão de obras. Em relação à utilização da gestão Lean, informou que essa abordagem tem sido muito utilizada na empresa para a otimização dos processos de construção, tendo tido resultados positivos no canteiro de obras. A utilização do controle do cronograma com o LPS e o TTP está sendo introduzida na empresa com grande aceitação e ótimos resultados. O resumo do estudo de caso em relação ao uso das metodologias de gestão se encontra no Quadro 4.

Quadro 4 - Resumo do uso das Metodologias de Gestão

\begin{tabular}{|c|c|c|c|c|c|c|c|}
\hline Projeto & Empresa & País & Cronograma & Custo & EVM & Risco & Lean \\
\hline 1 & D & Brasil & $\checkmark$ & $\checkmark$ & X & X & X \\
\hline 2 & E & Brasil & $\checkmark$ & $\checkmark$ & $\checkmark$ & X & X \\
\hline 3 & F & Índia & $\checkmark$ & $\checkmark$ & X & X & X \\
\hline 4 & G & México & $\checkmark$ & $\checkmark$ & $\checkmark$ & X & X \\
\hline 5 & H & Ucrânia & $\checkmark$ & $\checkmark$ & X & X & X \\
\hline 6 & I & Alemanha & $\checkmark$ & $\checkmark$ & $\checkmark$ & $\checkmark$ & $\checkmark$ \\
\hline
\end{tabular}

Fonte: Os próprios autores.

No Quadro 4, encontra-se resumida a utilização das metodologias de gestão pelos projetos de cada empresa, estando assinalados com " $X$ " os casos em que a metodologia não é utilizada e com " $\checkmark$ " os casos em que é. Pode se observar que em todas as empresas entrevistadas a 
gestão do cronograma e custo é realizada. A gestão por EVM é realizada em três empresas, já a gestão de risco e Lean, em apenas uma empresa. Somente a empresa alemã utilizou todas as metodologias de gestão.

Em relação às hipóteses da pesquisa, foram identificados os seguintes aspectos. Foi verificado que as empresas " $D$ " e " $E$ " apresentaram dificuldades no acompanhamento do cronograma devido ao controle da execução das atividades no canteiro de obras. A empresa "E" teve dificuldade na utilização do EVM em virtude da necessidade de integração do cronograma e por causa da baixa maturidade em gestão de projetos. Foi verificado também que as empresas $D$, E, F, G e H utilizam as metodologias de gestão de maneira incompleta e apresentam deficiências no planejamento. Pode-se observar que apenas a empresa "I" utilizou a metodologia de gestão do PMBOK de maneira completa e a gestão enxuta, obtendo benefícios com a sua utilização.

\section{PROPOSTAS DE MELHORIAS NA GESTÃO DA CONSTRUÇÃO CIVIL}

De acordo com os resultados obtidos, os aspectos mais relevantes para a gestão foram categorizados em gestão de pessoas, tecnologia da informação, metodologia de gestão e a estrutura organizacional. Sob a perspectiva de gestão de pessoas, foi observado que o treinamento e a integração das equipes são os aspectos mais relevantes. O treinamento é importante, pois a maior parte das empresas não utiliza as ferramentas de gestão por falta de conhecimento da equipe de projeto. Outro aspecto relevante é a integração das diferentes equipes que trabalham no projeto. A liderança do gerente de projeto também é um aspecto de grande importância, pois esse promove a integração da equipe. Em relação à estrutura organizacional, foi observado que a gestão "projetizada" (estrutura organizacional baseada em projetos) é a mais adequada ao controle e à gestão de projetos de construção. Cabe destacar, ainda, a importância da utilização de sistemas de TI, que apoiam o uso das metodologias de gestão. Verificou-se que a utilização de sistemas isolados de TI dificulta a integração das informações e das diferentes equipes.

No Quadro 5, encontram-se resumidas as melhorias propostas em relação a pessoas, estrutura organizacional e tecnologia da informação.

Quadro 5 - Propostas de melhorias na gestão da construção civil

\begin{tabular}{|c|l|l|}
\hline Item & \multicolumn{1}{|c|}{ Descrição } & \multicolumn{1}{c|}{ Propostas de melhorias } \\
\hline 1 & Pessoas & $\begin{array}{l}\text { Integração da equipe, liderança do gerente de projeto e o } \\
\text { treinamento da equipe em gestão. }\end{array}$ \\
\hline 2 & Estrutura Organizacional & $\begin{array}{l}\text { Estrutura organizacional baseada em projetos (gestão } \\
\text { "projetizada") que melhora o controle e a gestão do } \\
\text { empreendimento. }\end{array}$ \\
\hline 3 & Tecnologia da Informação & $\begin{array}{l}\text { Utilização de sistemas de TI que apoiam o uso das } \\
\text { metodologias de gestão, automatizando os processos e } \\
\text { aumentando a confiabilidade nas informações. As empresas } \\
\text { devem buscar utilizar sistemas integrados de gestão. }\end{array}$ \\
\hline
\end{tabular}

Fonte: Os próprios autores. 


\section{CONCLUSÃO}

A indústria da construção tem um forte impacto na economia local, entretanto problemas de gestão comprometem o desempenho dessas empresas. Foram feitas entrevistas em projetos de construção do Brasil, Índia, México, Ucrânia e Alemanha para avaliar a gestão da construção. Foi verificado que a maior parte das empresas realiza a gestão das obras utilizando cronograma e planilhas de custo, comprometendo a gestão das obras e o controle total do projeto. Verificouse que, em sua maior parte, esse fato se deve à deficiência no conhecimento de metodologias de gestão, a falhas no planejamento de obras e à baixa qualificação da equipe de gestão. Foi observado que as empresas privadas, maiores e com maior treinamento, utilizam as ferramentas de gestão de maneira mais adequada.

Em relação às propostas de melhorias, elas foram subdivididas em: pessoas, estrutura da empresa, utilização da tecnologia da informação, metodologias e processos de gestão. Em relação às pessoas, foi verificada a necessidade de treinamento e integração entre as diferentes equipes da obra. Sob o aspecto da estrutura da empresa, foi verificado que a estrutura orientada a projetos ("projetizada") apresenta melhores resultados. A utilização de ferramentas de TI é importante, pois elas automatizam os processos de gestão e possibilitam a integração das informações e pessoas.

Portanto os aspectos destacados por diferentes autores sobre os problemas na gestão da construção, como falhas de planejamento, informações incompletas sobre custos, falhas no controle de execução, falta de suporte da alta administração, falta de treinamento, falta de maturidade de gestão de projetos e ausência de um software integrado de gestão, foram confirmados nesta pesquisa e comprometem o prazo e o custo dos projetos de construção. As empresas de construção devem aperfeiçoar os seus sistemas de gestão para que possam atender o desenvolvimento local e contribuir para o crescimento do PIB.

\section{AGRADECIMENTOS}

Ao Prof. Bertram Lomüller, Phd da Steinbeis Global Institute, pela oportunidade de desenvolver a pesquisa na cidade de Tübingen, na Alemanha.

\section{REFERÊNCIAS}

ANDÚJAR-MONTOYA, M. D.; GILART-IGLESIAS, V.; MONTOYO, A.; MARCOS-JORQUERA, D. A construction management framework for mass customisation in traditional construction. Sustainability, v. 7, n. 5, p. 1-29, 2015.

ARROTÉIA, A. V.; AMARAL, T. G.; MELHADO, S. B. Gestão de projetos e sua interface com o canteiro de obras sob a ótica da Preparação da Execução de Obras. Ambiente Construído, Porto Alegre, v. 14, n. 4, p. 183-200, 2014.

BABAR, S.; THAHEEM, M. J.; AYUB, B. Estimated cost at completion: integrating risk into earned value management. Journal of Construction Engineering and Management, v. 143, n. 3, p. 04016104-104016104-11, mar. 2017.

DANIEL, E. I.; Pasquire, C.; Dickens, G.; Ballard, H. G. The relationship between the Last Planner ${ }^{\circ}$ System and collaborative planning practice in UK construction. Engineering, Construction and Architectural Management, v. 24, n. 3, p. 407-25, 2017 
DE FILIPPI, G. A.; MELHADO, S. B. Um estudo sobre as causas de atrasos de obras de empreendimentos imobiliários na região Metropolitana de São Paulo. Ambiente Construído, Porto Alegre, v. 15, n. 3, p. 161-73, 2015.

DOBBS, R.; POHL, H.; LIN, D. Y. Infrastructure productivity: how to save \$1 trillion a year. McKinsey Global Institute. [S.I.]: Print, 2013.

FARROKHI, F.; MAHMOUDI-HAMIDABAD, A. Rethinking convenience sampling: Defining quality criteria. Theory and practice in language studies, v. 2, n. 4, p. 784, 2012.

FIRJAN. Construção Civil: desafios 2020. Rio de Janeiro, 2014. Disponível em: http://www.firjan.com.br/ lumis/portal/file/fileDownload.jsp?fileld=4028808B4E3FB673014E3FF18D3D20D8. Acesso em: 15 set. 2017.

FRANDSON, A.; BERGHEDE, K.; TOMMELEIN, I. D. Takt time planning for construction of exterior cladding. In: CONFERENCE OF THE INTERNATIONAL GROUP FOR LEAN CONSTRUCTION, 21., Fortaleza, 2013. Anais [...], Fortaleza: IGLC, 2013.

FREITAG, A. E. B. Fatores críticos de sucesso para adoção da gestão "enxuta" pela indústria da construção civil do estado do Rio de Janeiro. Orientador: Osvaldo Luiz Gonçalves Quelhas. 2015. Tese (Doutorado em Engenharia Civil) - Universidade Fluminense, Niterói, RJ, 2015.

GIL, A. C. Como elaborar projetos de pesquisa. 4.ed. São Paulo: Atlas, 2002.

GRAU, D.; ABBASZADEGAN, A.; TANG, P., GANAPATHY, R., DIOSDADO, J. A combined planning and controls approach to accurately estimate, monitor, and stabilize work flow. In: INTERNATIONAL CONFERENCE ON COMPUTING IN CIVIL AND BUILDING ENGINEERING, 24., Computing in Civil and Building Engineering. 23-25 jun. 2014, Orlando, Florida. Anais [...]. American Society of Civil Engineers (Asce), 2014. p. 105-12.

HORSTMAN, A.; WITTEVEEN, W. Performance indicators in the best value approach. Journal for the Advancement of Performance Information \& Value, v. 5, n. 2, 2013.

KIM, Y.-W.; BALLARD, G. Management thinking in the earned value method system and the last planner system. Journal of Management in Engineering, v. 26, n. 4, p. 223-28, 2010.

KOSKELA, L.; HOWELL, G.; PIKAS, E.; DAVE, B. If CPM is so bad, why have we been using it so long? In: THE 22TH INTERNATIONAL GROUP FOR LEAN CONSTRUCTION CONFERENCE, 2327 jun. 2014, Oslo, Norway. Anais [...]. University of Huddersfield, 2014.

LOWE, R. H.; D'ONOFRIO, F. M.; FISK, M. D.; SEPPÄNEN, O. A comparison of location-based scheduling with the traditional critical path method. In: ANNUAL MEETING AMERICAN COLLEGE OF CONSTRUCTION LAWYERS, 2012, San Francisco. Anais [...]. San Francisco: [s.n], 2012.

MELO, M.; DESCHAMPS, F.; DA COSTA, S. E. G. Application of Lean Construction - a Systematic Literature Review. Journal of Lean Systems, v. 2, n. 3, 2017.

MOURA, C. B.; FORMOSO, C. T. Análise quantitativa de indicadores de planejamento e controle da produção: impactos do Sistema Last Planner e fatores que afetam a sua eficácia. Ambiente Construído: Revista da Associação Nacional de Tecnologia do Ambiente Construído, Porto Alegre, v. 9, n. 3, p. 57-74, jul./set. 2009.

NARBAEV, T.; DE MARCO, A. Combination of growth model and earned schedule to forecast project cost at completion. Journal of Construction Engineering and Management, v. 140, n. 1, p. 04013038, 2013. 
NASIRZADEH, F.; KHANZADI, M., REZAIE, M. Modelagem dinâmica da alocação quantitativa de risco em projetos de construção. Jornal internacional de gerenciamento de projetos, Amsterdã, v. 32, p. 442-51, 2014.

NAZÁRIO, L.; AZEVEDO, E. Gestão de projetos na construção civil em Natal-estudo exploratório no mercado imobiliário. In: CONGRESSO INTERNACIONAL DE ADMINISTRAÇÃO, 2016, Natal. Anais [...] , 2016. p. 1-90. http://www.admpg.com.br/2016/down.php?id=2324\&q=1. Acesso em: 15 set. 2017.

NETTO, J. T.; QUELHAS, O. L. G.; FRANÇA, S.; MEIRINO, M. J. Estudo comparativo entre as práticas empresariais e a teoria de gerenciamento por Valor Agregado: o caso da construção civil. Ambiente Construído, Porto Alegre, v. 15, n. 3, p. 145-60, set. 2015.

OLIVIERI, H.; GRANJA, A. D.; PICCHI, F. A. Planejamento tradicional, Location-Based Management System e Last Planner System: um modelo integrado. Ambiente Construído, v. 16, n. 1, p. 265-83, 2016.

PROJECT MANAGEMENT INSTITUTE. Guia PMBOK ${ }^{\circledR}$ : um guia para o conjunto de conhecimentos em gerenciamento de projetos. 6. ed. Pennsylvania: PMI, 2017.

RENUKA, S. M.; UMARANI, C.; KAMAL, S. A review on critical risk factors in the life cycle of construction projects. Journal of Civil Engineering Research, v. 4, n. 2A, p. 31-6, 2014.

SEPPÄNEN, O.; EVINGER, J.; MOUFLARD, C. Effects of the location-based management system on production rates and productivity. Construction management and economics, v. 32, n. 6, p. 608-24, 2014.

ZHANG, Y. A framework to improve modular construction manufacturing production line performance. [s.l.]: University of Alberta, 2017.

\section{Sobre os autores:}

Joaquim Teixeira Netto: Doutor e mestre em Engenharia Civil pela Universidade Federal Fluminense (UFF). Especialista em Análise de Sistemas pela Universidade Estácio de Sá (Unesa). Graduado em Engenharia Mecânica pela Universidade Federal do Rio de Janeiro (UFRJ) e certificado Project Management Professional (PMP). Pesquisador da Fiocruz na Escola Nacional de Saúde Pública (Ensp), no Centro de Saúde Escola Germano Sinval Faria (CSEGSF), onde é coordenador adjunto do Laboratório Internet Saúde e Sociedade (LaISS). E-mail: joaquimtnetto@gmail.com, Orcid: http://orcid.org/0000-0002-7392-9166

João Alberto Neves: Doutor em Engenharia de Produção pela Pontifícia Universidade Católica do Rio de Janeiro (PUC-Rio). Mestre em Sistemas e Computação e graduado em Engenharia Mecânica pelo Instituto Militar de Engenharia. Professor da Faculdade de Administração e Ciências Contábeis, e dos Programas de Pós-Graduação em Administração e em Engenharia Civil da Universidade Federal Fluminense (UFF). E-mail: joaoalbertoneves@gmail.com, Orcid: http://orcid.org/0000-0002-4812-6214

Walter Passos Filho: Graduado em Engenharia Elétrica pela Universidade Veiga de Almeida. Experiência na área de Engenharia Elétrica, com ênfase em Engenharia Elétrica. E-mail: wpassosfilho@gmail.com, Orcid: http://orcid.org/0000-0001-5908-020X

Nylvandir Liberato Oliveira: Doutorando em Engenharia Civil pela Universidade Federal Fluminense (UFF). Mestre em Ciências Contábeis pela Universidade do Estado do Rio de Janeiro. 
Graduado em Economia e em Ciências Contábeis pela Universidade Estácio de Sá (Unesa). Professor adjunto da Universidade Estácio de Sá e da Escola Superior Nacional de Seguros (ESNS/ Funenseg). E-mail: liberato.finan@gmail.com, Orcid: http://orcid.org/0000-0002-7063-0556 